\title{
Compositional Fractionation of Copoly(styrene/methyl methacrylate) by Normal-Phase and Reversed-Phase High-Performance Liquid Chromatography
}

\author{
Shinya Teramachi, Akira Hasegawa, and Ken Motoyama \\ Department of Industrial Chemistry, Kogakuin University, \\ Nakano-cho 2665-1, Hachioji, Tokyo 192, Japan
}

(Received December 15, 1989)

\begin{abstract}
It was studied whether both modes of normal-phase (NP) and reversed-phase (RP) of adsorption chromatography can work effectively for the compositional fractionation of copolymer by high-performance liquid chromatography (HPLC) under the coexistence of both mechanisms of adsorption and phase-separation. The fractionation of a mixture of 4 copoly(styrene/methyl methacrylate) samples with different chemical compositions by gradient HPLC was tried for 8 cases using combinations of 2 eluents of tetrahydrofuran(THF)/cyclohexane $(\mathrm{CHX})$ and THF/acetonytrile(ACN), and 4 columns of modified silica gel with $\mathrm{NH}_{2^{-}}, \mathrm{CN}-$, Phenyl- and octadecyl(ODS)-groups. The mixture was fractionated effectively according to chemical composition by 4 combinations of THF/CHX and high-polar columns $\left(\mathrm{NH}_{2}, \mathrm{CN}\right)$, and THF/ACN and non-polar columns (Phenyl, ODS), which are the fitting combinations for the scheme of NP or RP adsorption. However, other combinations, could not give the effective separation. For the gradient HPLC by the fitting combinations, THF-contents of the eluents at peak positions were higher than those at the first cloud points for the respective copolymer samples, and also the molecular weight dependence of the peak position was negligible. It may thus be concluded that adsorption mechanism is predominant than phase-separation mechanism in the system of the fitting combination of eluents and columns for the scheme of NP or RP mode.
\end{abstract}

KEY WORDS Compositional Fractionation / High-Performance Liquid Chromatography / Normal-Phase Adsorption / Reversed-Phase Adsorption / Phase-Separation / Copoly(styrene/methyl methacrylate) /

For the molecular characterization of copolymers, it is important to develop automatic methods to determine the chemical composition distribution (CCD). High-performance liquid chromatography (HPLC) is powerful and an automatic method for separation analysis. Although HPLC has been applied for the analysis of low molecular weight compounds by various mechanisms such as adsorption, partition, ion-exchange, gel-permeation or size-exclusion and so on, only gel-permeation chromatography (GPC) has been applied for analysis of synthetic polymers among the mechanisms for a long time. In 1978, however, HPLC was applied to the compositional fractionation of copoly(styrene/methyl acrylate) at first. ${ }^{1,2}$ Since then, several authors have applied HPLC for this purpose ${ }^{3-25}$ and a comprehensive review on the subject was also published. ${ }^{26}$

The adsorption-desorption mechanism has been applied for copolymer HPLC since the first application, while phase-separation or selective precipitation-dissolution mechanism was applied as "HP-precipitation-LC" by Glöckner et al. ${ }^{5}$ Also in the system of Danielewicz and Kubin, ${ }^{4}$ it is certain that phase-separation occurs, though they wrote "adsorption chromatography" in their paper. Both mechanisms of adsorption-desorption and phase-separation are involved in the compositional fractionation of copolymers by 
HPLC, if the CCD of the copolymer sample covers a broad range and the difference between polarities of the respective monomeric units is not small. For such copolymer samples, the eluent composition ranges of dissolution limit and desorption limit should be broad and then both ranges should overlap one another, as shown in Figure 8.

For the analysis of low molecular weight compounds by adsorption chromatography, it is well known that there are two modes of normal-phase (NP) and reversed-phase (RP). The eluent composition is changed from low polarity to high polarity on high-polar column in the former mode, but from high polarity to low polarity on non-polar column in the latter mode. For the separation of synthetic copolymers, recently, not only the NP mode, which had been used exclusively in the early works, but also the RP mode were applied successfully. ${ }^{16,17,23}$

The solubilities of eluents for copolymer samples are determinative in the phaseseparation mechanism which has been used in classical compositional fractionations. ${ }^{27-29}$ If monomeric units $A$ and $B$ in copoly(A/B) are polar and non-polar or high and low-polar, respectively, high-polar solvents may be good for copolymer components of high A-content, while low-polar solvents may be good for components of low A-content. In HPLC by phase-separation mechanism, the gradient elution from low-polarity to high-polarity should elute out the copolymer sample from the components of low A-content to those of high A-content, whereas the gradient elution with the reverse direction should elute out the sample from the components of high A-content to those of low A-content.

Thus, the directions of gradient elutions by both mechanisms of adsorption and phaseseparation for compositional fractionation should be in agreement with one another for copolymers composed of monomeric units of low- and high-polarities. Therefore, both modes of adsorption chromatography (NP and
RP) may work effectively in general under the condition of coexistence of both mechanisms. It is the purpose of the present work to study the compositional fractionation of copoly(styrene/methyl methacrylate), by both NP and RP columns under the condition of coexistence of both phase-separation and adsorption mechanisms.

\section{EXPERIMENTAL}

\section{Materials}

The copolymer samples were styrene(S)methyl methacrylate(MMA) statistical copolymers prepared in bulk at $60^{\circ} \mathrm{C}$ using benzoyl peroxide as the initiator. S-contents obtained by elemental analysis, number-average molecular weights by osmomentry and weight conversions of these samples are summerized in Table I. The fractions with different molecular weights shown in Table II were also used which were obtained by successive precipitation fractionation of sample SMMA81-50 using 2-butanone as solvent and isopropyl ether as precipitant at $30^{\circ} \mathrm{C}$. The solvents used for HPLC, tetrahydrofuran (THF), cyclohexane ( $\mathrm{CHX})$, and acetonytrile

Table I. The samples of SMMA

\begin{tabular}{cccc} 
Sample & S/mol\% & $M_{n} \times 10^{-5}$ & Conv. $/ \mathrm{wt} \%$ \\
\hline SMMA-8-25 & 23.1 & 1.22 & 32.8 \\
$-81-50$ & 48.7 & 1.27 & 17.0 \\
-55 & 53.7 & $(1.50)$ & 2.0 \\
-75 & 74.5 & 1.88 & 2.9 \\
$-81-75$ & 85.4 & 1.40 & 10.9 \\
\hline
\end{tabular}

( ), presumed value from viscosity data.

Table II. The fractions of SMMA-81-50

\begin{tabular}{cc}
\hline Fraction No. & $M_{n} \times 10^{-5}$ \\
\hline 1 & 4.33 \\
4 & 2.76 \\
5 & 2.45 \\
6 & 1.96 \\
8 & 0.90 \\
\hline
\end{tabular}


(ACN) were the chromatography class of Wako Pure Chemical Industries, Ltd. (Tokyo).

\section{Selection of Eluents}

Copolymer samples with different chemical compositions were dissolved in THF, which has modelate polarity, at the concentration of $0.01 \mathrm{~g} \mathrm{~cm}^{-3}$. The first cloud points were determined by titrating the solution with nonpolar and high-polar precipitants visually. These solvents must be transparent to ultraviolet light of the detector for HPLC. THF/CHX and THF/ACN were selected as the eluents for HPLC experiments, since solvent compositions at the first cloud points were largely depending upon the chemical compositions of the copolymer samples in these systems, as shown in Table III.

\section{Columns for HPLC}

Four columns were used for HPLC. $\mathrm{NH}_{2}$ columns ( $\mu$-Bondapak- $\mathrm{NH}_{2}$ (Waters Associates, U.S.A.), inner diameter; $\phi=3.9 \mathrm{~mm}$, length; $L=30 \mathrm{~cm}$, partile diameter; $d=5 \mu \mathrm{m}$ ) and $\mathrm{CN}$-column ( $\mu$-Bondapak-CN, $\phi=3.9$ $\mathrm{mm}, L=30 \mathrm{~cm}, d=5 \mu \mathrm{m}$ ) were used as the high polar columns. Phenyl-column ( $\mu$-Bondaspher $5 \mu$ Phenyl-100A $, \phi=3.9 \mathrm{~mm}, L=15 \mathrm{~cm}, d=$ $5 \mu \mathrm{m}$ ) and ODS (octadecyl)-column (TSK gel ODS-120T (Tosoh Corporation, Tokyo), $\phi=$ $4.6 \mathrm{~mm}, L=25 \mathrm{~cm}, d=5 \mu \mathrm{m}$ ) were used as the nonpolar columns.

\section{Instruments of HPLC}

The HPLC instrument was a Model HLC-802 (Tosoh Corporation), in which the pump was replaced with a high pressure pump of Type-510 (Waters Associates). The gradient controller was Type-680 (Waters Associates). The gradient controller was Type-510 (Waters Associates). The UV-detector was ERC-7211 (Erma Inc., Tokyo).

\section{Experimental Conditions}

The sample injected in HPLC was a equiweight mixture of 4 samples of SMMA-
Table III. First cloud points of THF solutions of SMMA samples by $\mathrm{CHX}$ and $\mathrm{ACN}$ as precipitant

\begin{tabular}{ccc}
\hline \multirow{2}{*}{ Samples } & \multicolumn{2}{c}{ vol\% of precipitant } \\
\cline { 2 - 3 } & CHX & ACN \\
\hline SMMA-81-25 & 49.4 & 85.6 \\
$-81-50$ & 65.9 & 70.8 \\
-55 & 68.0 & - \\
$-81-75$ & 88.2 & 57.4 \\
\hline
\end{tabular}

81-25, SMMA-81-50, SMMA-75 and SMMA81-75 dissolved in THF at the total concentration of $1.2 \mathrm{mg} \mathrm{cm}^{-3}$. For the fractions used for the study of molecular weight dependency, the concentration was $0.3 \mathrm{mg} \mathrm{cm}^{-3}$. The injection volumn was $0.1 \mathrm{~cm}^{3}$ in all measurements. The flow rate was $1.0 \mathrm{~cm}^{3} \mathrm{~min}^{-1}$. The column temperature was $30^{\circ} \mathrm{C}$. The wave length of UV-detector was $259 \mathrm{~nm}$. The eluent composition changed linearly from the start composition of low THF-content to the final composition of high THF-content for $15 \mathrm{~min}$. After the flow of the final composition for $5 \mathrm{~min}$, the eluent composition was changed to $100 \%$ THF. Pure THF flowed for $5 \mathrm{~min}$ and then the eluent composition was reduced to the start composition by linear gradient for 20 min. The start and the final compositions are shown in figure captions, respectively.

\section{RESULTS}

The fractionation of a mixture of 4 copolymer samples by HPLC was attempted for 8 cases. That is, combinations of 2 eluent systems of THF/CHX and THF/ACN, and 4 columns of $\mathrm{NH}_{2}, \mathrm{CN}$, Phenyl, and ODS, were used.

However, the mixture of SMMA samples was effectively fractionated according to chemical composition only in 4 cases. That is, the systems of $\mathrm{THF} / \mathrm{CHX}$ on high-polar columns $\left(\mathrm{NH}_{2}\right.$ and $\left.\mathrm{CN}\right)$ and the systems of THF/ACN on nonpolar columns (Phenyl and ODS) gave chromatograms of 4 peaks 


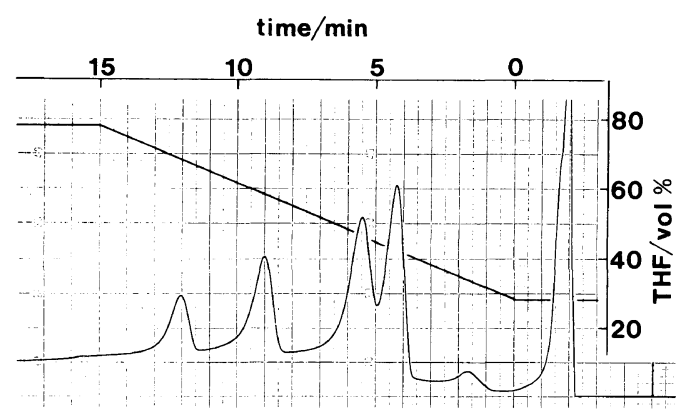

Figure 1. The chromatogram of a mixture of 4 samples of SMMA obtained by $\mathrm{NH}_{2}$-column and THF/CHX gradient elution from 28 to $78 \mathrm{vol} \%$ of THF. The straight line shows the gradient protocol.

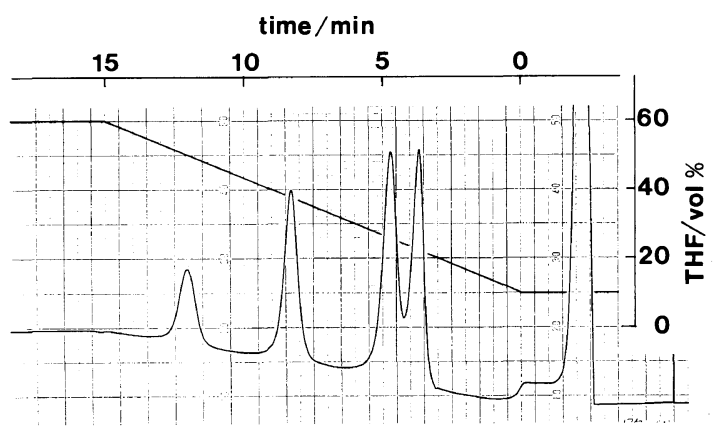

Figure 2. The chromatogram of a mixture of 4 samples of SMMA obtained by $\mathrm{CN}$-column and THF/CHX gradient elution from 10 to $60 \mathrm{vol} \%$ of THF. The straight line shows the gradient protocol.

corresponding to the original 4 samples, as shown in Figures $1-4$.

The systems of THF/ACN on high polar columns and the THF/CHX on nonpolar columns could not separate the mixture according to chemical composition. A chromatogram obtained by the system of $\mathrm{THF} /$ ACN on $\mathrm{NH}_{2}$-column is shown in Figure 5 as an example of chromatograms by such systems.

For 4 cases in which compositional fractionation was carried out effectively, the molecular weight dependence of the elution time was studied by the same gradient elutions as those in the respective cases, using the fraction of SMMA-81-50. The molecular weight dependency was negligible in every case as

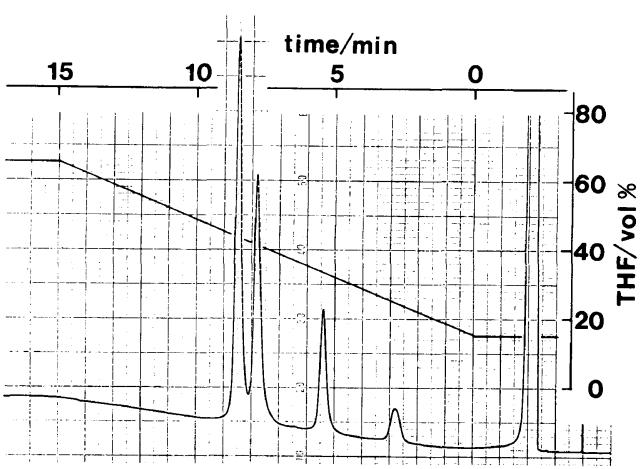

Figure 3. The chromatogram of a mixture of 4 samples of SMMA obtained by Phenyl-column and THF/ACN gradient elution from 15 to $65 \mathrm{vol} \%$ of THF. The straight line shows the gradient protocol.

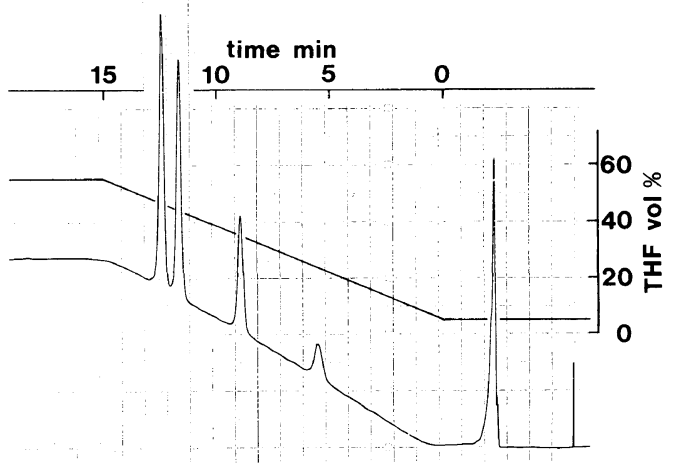

Figure 4. The chromatogram of a mixture of 4 samples of SMMA obtained by ODS-column and THF/ACN gradient elution from 5 to $55 \mathrm{vol} \%$ of THF. The straight line shows the gradient protocol.

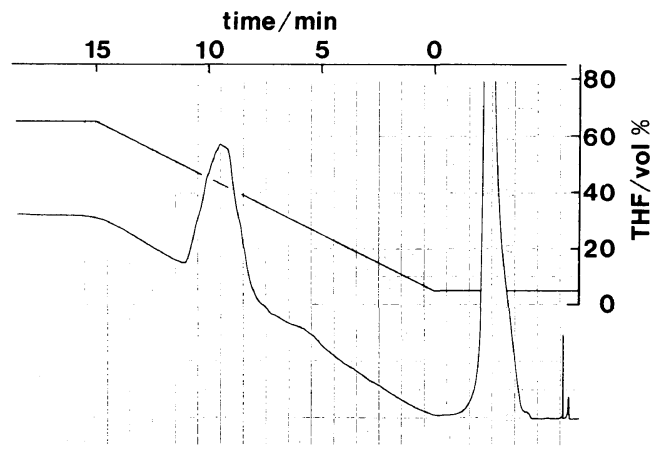

Figure 5. The chromatogram of a mixture of 4 samples of SMMA obtained by $\mathrm{NH}_{2}$-column and THF/ACN gradient elution from 5 to $65 \mathrm{vol} \%$ of THF. The straight line shows the gradient protocol. 
Table IV. Molecular weight dependency of THF-content of the eluent at the peak of SMMA-81-50 fractions

\begin{tabular}{ccccc}
\hline \multirow{4}{*}{ Fr. No. } & \multicolumn{5}{c}{ THF/vol\% } \\
\cline { 2 - 5 } & $\mathrm{NH}_{2}$ & CN & Phenyl & ODS \\
\hline 1 & 57.4 & 37.7 & 31.2 & 30.3 \\
4 & - & - & - & 30.2 \\
5 & 57.6 & 37.9 & 31.2 & - \\
6 & 57.4 & 37.7 & 31.1 & - \\
8 & 57.6 & 37.8 & 30.8 & 30.4 \\
\hline
\end{tabular}

shown in Table IV, in the range of molecular weight tested $\left(0.9-4.33 \times 10^{5}\right)$.

The reproducibility of the peak position was tested several times for the respective systems which gave good compositional separation. The elution times or the eluent compositions at the respective peaks were in good agreement with one another in all cases. Differences in the data were $0.2-0.3 \mathrm{~min}$ in the elution time or about $0.6 \mathrm{vol} \%$ in THF-content, at most.

\section{DISCUSSION}

From the point of view of the phaseseparation mechanism, it is clear from the data in Table III (and also Figures 6 and 7) that the eluent of THF/CHX may elute out the present copolymer from the components of high S-content to those of low S-content, while the eluent of THF/ACN may elute out the samples from the components of high MMA-content to those of low MMA-content, irrespective of kinds of the column used.

Actually, however, effective compositional fractionation was attained in limited cases. That is, only the systems of $\mathrm{THF} / \mathrm{CHX}$ on high-polar columns and THF/ACN on nonpolar columns, of which results were shown in Figures 1-4, gave effective compositional fractionations. These systems are combinations of eluents and columns fitting the scheme of NP or RP adsorption. Effective fractionation could not be attained by non-fitting combina-

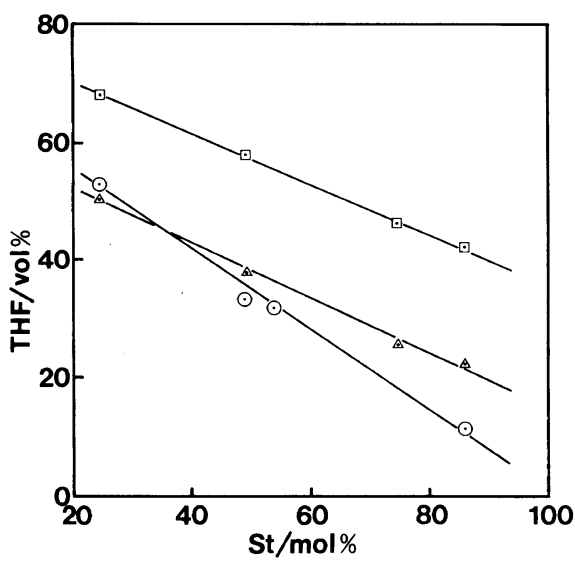

Figure 6. Relationships between S-contents of SMMA samples and THF-contents at the first cloud points $(\odot)$, peak positions of the chromatogram by $\mathrm{NH}_{2}$-column (Figure 1, $\square$ ) and those by $\mathrm{CN}$-column (Figure 2, $\triangle$ ) for the elutent system of THF/CHX.

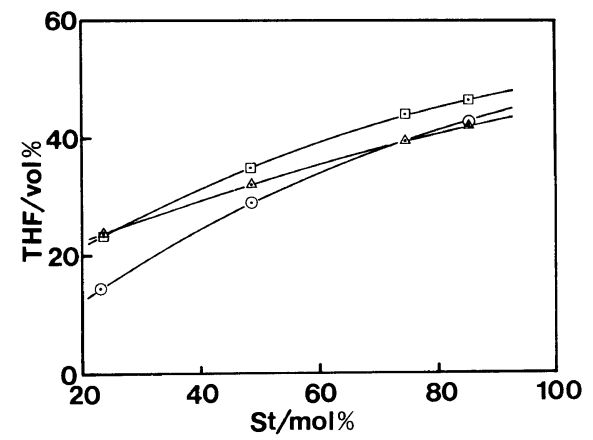

Figure 7. Relationships between S-contents of SMMA samples and THF-contents at the first cloud points $(\odot)$, peak positions of the chromatogram by Phenyl-column (Figure $3, \triangle$ ) and those by ODS-column (Figure $4, \square$ ) for the eluent system of THF/ACN.

tions for the scheme, as an example of the results was shown in Figure 5.

These results are consistent with the results of Glöckner et al. and Sato et al. It was reported by Glöckner et al. that copoly(styrene/ethyl methacrylate) was fractionated effectively by the fitting combinations for NP (THF/isooctane on silica) and RP (THF/methanol on ODS) modes, respectively, but the separation by the non-fitting combination of NP eluent and RP column (THF/iso-octane on ODS) was 
poor for the copolymer, ${ }^{17}$ and also effective fractionation of copoly(S/MMA) was attained by fitting combination for NP mode (THF/isooctane on silica) but not by non-fitting combinations (THF containing 10\% methanol $/ n$-hexane or iso-octane on $\mathrm{C}_{8}$ or $\mathrm{C}_{18^{-}}$ column). ${ }^{16}$ Sato et al. demonstrated that the fitting combination of RP mode (dichloromethane/ACN on cross-linked PS gel) gave compositional separation to copoly(S/MMA), effectively. ${ }^{23}$

In Figures 6 and 7, the eluent compositions at the first cloud points and at peak positions of the chromatograms are plotted against the chemical compositions of the copolymer samples. The THF-content at the peak position is higher than that at the first cloud point, compared at the same copolymer composition. (The differences between both points should be much larger than those in the figures, if taking into account that the polymer concentrations at the first cloud points are higher by about two orders than those in HPLC column.) This may show that the adsorption-desorption mechanism must work following selective dissolution.

As shown in Table IV, moreover, the molecular weight dependency of peak position was negligible for these systems. This means that adsorption mechanism is more predominant than phase-separation mechanism in these systems, since the molecular weight dependency is unavoidable in phase-separation mechanism, but is negligible for adsorption mechanism in the range of high molecular weight.

Precipitations could not occur in the eluents at the start compositions for the samples of SMMA-75 and SMMA-81-75 in the case of THF/CHX and $\mathrm{NH}_{2}$-column, as clear from Figure 6 and the gradient range shown in Figure 1, and for SMMA-81-25 in the case of THF/ACN and Phenyl-column as clear from Figures 7 and 3. Thus possibly, these components were not fractionated by phaseseparation mechanism but separated only by adsorption mechanism.

It may thus be concluded that the adsorption mechanism is more predominant than phaseseparation mechanism in the systems in which the compositional fractionation of SMMA copolymer was attained effectively. Nevertheless, the importance of the phase-separation mechanism should not be neglected under the coexistence of adsorption and phase-separation phenomena. It should be rather considered that the effective compositional fractionations shown in Figures $1-4$ were attained by the co-operation of both mechanisms.

By non-fitting combinations of eluents and columns for the schemes of NP and RP adsortpion, the present copolymer samples were not fractionated effectively. Also in these cases however, it is supposed from the data of the first cloud points that the phase-separation mechanism works effectively. In phase-separation, the effect of molecular weight is unavoidable. Therefore, the widths of peaks obtained by phase-separation mechanism should be broad according to the molecular weight distributions (MWD) of the samples. Thus, the peaks of samples which have broad MWD's as the present sample should overlap one another.

In addition to the effect of MWD, the interactions between the copolymer components dissolved and surfaces of the packing materials in the columns may work in the opposite direction to compositional fractionation. For example, in THF/CHX, components with high S-content should be dissolved first, but interactions between the components and the surfaces of nonpolar packing materials (ODS or Phenyl) may be attractive, compared with the interactions between the components with high MMA-content dissolved later and the suface. On the other hand, the components with high MMA-content should be dissolved later by the eluent of high THF-content, but the interactions between the components and the nonpolar surface may be repulsive. It may thus be speculated that the copolymer 
components separated by selective dissolution may be mixed again, depending upon balance among three interactions between eluent and copolymer component, copolymer coponent and surface, and also surface and eluent.

On the other hand, it was demonstrated by Glöckner et al. that the good separation of copoly(styrene/acrylonitril) was achieved not only by fitting combinations for NP adsorption but also by non-fitting combinations of NP eluent and RP column. ${ }^{16,24}$ For the relationship between the eluent composition and copolymer composition in their work, the peak position and first cloud point were in agreement with one another. This means that only phase-separation mechanism was effective on the fractionation of the copolymer, but adsorption mechanism had no effect on the fractionation.

We can suppose the seheme shown in Figure 8 for the separation of copolymers composed of polar and nonpolar or high and low-polar monomeric units. Although the soluble band is essentially determined by balance of solubility parameters of the eluent and the copolymer, the solubility parameter may change roughly parallel to the polarity for such copolymers. On the other hand, the position of the adsorption limit may depend upon balance among the polarities of copolymer components, eluents and adsorbents. If the adsorption limit lines are inside the soluble band (chain lines), adsorption mechanism may be predominant, while only phase-separation mechanism may be effective if the imaginary adsorption limit lines are outside the soluble band (dashed lines). If the polarity of copolymer components is very high, the condition of the former case may scarcely come about.

The present results are the former case shown in Figures 6 and 7, while the results of copoly(styrene/acrylonitrile) by Glöchner et al. may be the latter case since the polarity of acrylonitrile monomeric unit is very high. Further investigation is needed to establish

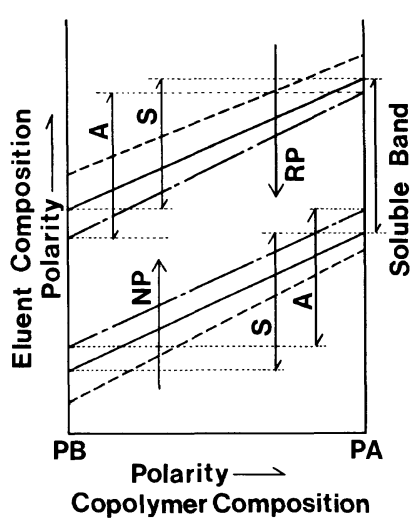

Figure 8. Schematic relationship between copolymer composition and eluent composition for HPLC separation of copolymer. - , dissolution limit; - --, desorption limit; ---, imaginary desorption limit; A, range of desorption limit; S, range of dissolution limit; NP, direction of normal-phase gradient elution; RP, direction of reversedphase gradient elution.

whether the scheme is effective in general.

In the separation of low molecular weight compounds by ODS-column, the adsorption is not necessarily considered the main mechanism, but partition should be taken into account. Therefore, we would not exclude partition from the separation mechanism, though the separation mechanism by the interaction with the packing material discussed only in terms of adsorption in this paper.

Acknowledgements. The present work was supported by a Grant-in-Aid for Scientific Research from the Ministry of Education, Science, and Culture of Japan. A part of the contents of this paper was reported at SPSJ 36th Polymer Symposium, Tokyo, Japan, October 21-24, 1987.

\section{REFERENCES}

1. S. Teramachi, A. Hasegawa, Y. Shima, and M. Akatsuka, Polym. Prepr., Jpn., 27, 560 (1978).

2. S. Teramachi, A. Hasegawa, Y. Shima, M. Akatsuka, and M. Nakajima, Macromolecules, 12, 992 (1979).

3. M. Danielewicz and M. Kubin, J. Appl. Polym. Sci., 26, 951 (1981). 
4. M. Danielewicz, M. Kubin, and S. Vozka, J. Appl. Polym. Sci., 27, 3629 (1982).

5. G. Glöckner, H. Kroschwitz, and Ch. Meissner, Acta Polymerrica, 33, 614 (1982).

6. G. Glöckner, Pure Appl. Chem., 55, 1553 (1983).

7. G. Glöckner and R. Koningsveld, Makromol. Chem., Rapid Commun., 4, 529 (1983).

8. G. Glökner, Jo H. M. van den Berg, N. L. J. Meijerink, T. G. Scholte, and R. Koningsveld, Macromolecules, 17, 962 (1984).

10. G. Glöckner, Jo H. M. van den Berg, N. L. J. Meijerink, T. G. Scholte, and R. Koningsveld, $J$. Chromatogr., 317, 615 (1984).

11. H. Sato, H. Takeuchi, S. Suzuki, and Y. Tanaka, Makromol. Chem., Rapid Commun., 5, 719 (1984).

12. G. Glöckner and Jo H. M. van den Berg, Chromatographia, 19, 55 (1984).

13. H. Sato, H. Takeuchi, and Y. Tanaka, Macromolecules, 19, 2613 (1986).

14. S. Mori, Y. Uno, and M. Suzuki, Anal. Chem., 58, 303 (1986).

15. G. Glöckner and Jo H. M. van den Berg, $J$. Chromatogr., 352, 511 (1986).

16. G. Glöckner and Jo H. M. van den Berg, $J$. Chromatogr., 384, 135 (1987).
17. G. Glöckner, J. Chromatogr., 403, 280 (1987).

18. G. Glöckner and Jo H. M. van den Berg, Chromatographia, 24, 233 (1987).

19. S. Mori and Y. Uno, J. Appl. Polym. Sci., 34, 2689 (1987).

20. S. Mori and Y. Uno, Anal. Chem., 59, 90 (1987).

21. G. Glöckner, M. Stickler, and W. Wunderlich, Fresenius Z. Anal. Chem., 328, 76 (1987).

22. G. Glöckner, M. Stickler, and W. Wunderlich, Fresenius Z. Anal. Chem., 330, 46 (1988).

23. H. Sato, K. Mitsutani, I. Shimizu, and Y. Tanaka, J. Chromatogr., 447, 387 (1988).

24. G. Glöckner, J. Appl. Polym. Sci., Appl. Polym. Symp., 43, 39 (1989).

25. S. Mori, J. Appl. Polym. Sci., Appl. Polym. Symp., 43, 65 (1989).

26. G. Glöckner, Adv. Polym. Sci., 79, 159 (1986).

27. A. V. Topchiev, A. D. Litomanovich and V. Ya. Shtern, Dokl. Akad. Nauk SSSR, 147, 1389 (1962).

28. S. Teramachi and M. Nagasawa, J. Macromol. Sci. Chem., A2, 1169 (1968).

29. G. Riess and P. Callot, "Fractionation of Copolymer" in "Fractionation of Synthetic Polymers," L. H. Tung, Ed., Marcel Dekker, New York, N.Y., 1977. 